\section{Ocular alterations in alopecia areata}

\begin{abstract}
Purpose To determine the ocular alterations occurring in alopecia areata with regard to the lens and fundus.

Methods Seventy-five patients with alopecia areata were examined. Seventy healthy control patients unaffected by skin, ocular or systemic disorders were also studied.

Results Symptomless punctate lens opacities were found in $38(51 \%)$ patients, whereas only $2(3 \%)$ control patients had similar lens changes. Fundus alterations were found in $\mathbf{3 1}$ $(41 \%)$ cases of alopecia areata and in only 16 $(23 \%)$ controls.

Conclusions These ocular alterations and their prevalence are reported and some theories regarding the possible aetiopathogenetic mechanisms are discussed.
\end{abstract}

Key words Aetiopathogenetic mechanisms, Alopecia areata, Fundus alterations, Punctate lens opacities

Alopecia areata patients have asymptomatic, isolated areas of hair loss usually of the scalp, though any area of the body may be involved. Normal hair growth usually resumes after a variable period but at times spontaneous remission does not occur and loss of hair extends to all of the scalp (alopecia totalis) or the entire body (alopecia universalis). Many theories have been advanced for the obscure pathogenesis behind this condition, but recently there seems to be strong evidence for an autoimmune mechanism with mediation of $\mathrm{T}$ cells. $^{1}$

Ocular alterations have previously been reported in alopecia areata (AA). Indeed, there have been various reports of lens alterations, from cases of cataract ${ }^{2-4}$ to minor punctate opacities. ${ }^{5-7}$ However, there are contrasting opinions as to the prevalence of these lens changes. Involvement of the chorioretina has also been reported in $\mathrm{AA}^{5,8,9}$ but the significance and aetiopathogenesis of both the lens changes and the fundus alterations in patients with AA is still obscure. The purpose of
SANTI MARIA RECUPERO, SOLMAZ

ABDOLRAHIMZADEH, MARCO DE

DOMINICIS, ROBERTO MOLLO,

ISABELLA CARBONI, LUCREZIA ROTA, STEFANO CALVIERI

this study was to investigate the prevalence and appearance of the ocular changes occurring in AA.

\section{Patients and methods}

Seventy-five patients ( 25 men, 50 women; age range 7-61 years) with AA who were seen for dermatological reasons at the Dermatology Institute of the University of Rome 'La Sapienza' were referred for ophthalmological examination as part of the study and solely because of the presence of AA. All patients were subjected to the following tests: visual acuity, applanation tonometry, anterior segment biomicroscopy with study of the lens and fundus ophthalmoscopy after full pharmacological mydriasis. Seventy healthy control patients ( 25 men, 45 women; chosen to be comparable in age to the AA patients) unaffected by skin, ocular or systemic disorders were also studied. The cases of AA were divided into groups designated as: universalis (complete loss of scalp and body hair), totalis (complete loss of scalp hair) and areata (incomplete loss of the hair from the scalp, beard or other body areas).

\section{Results}

The personal history of various diseases in the AA patients is shown in Table 1. In 24 (32\%) there was thyroid pathology and in 17 (23\%) there was a history of atopic dermatitis. Two patients suffered from vitiligo, 3 had anaemia (microcytaemia), 3 were affected with diabetes mellitus, 1 had Down's syndrome and 15 patients $(20 \%)$ had neuropsychiatric problems. The patients in the control group were unaffected by skin, ocular or systemic disorders. Even though they were chosen to be of ages comparable to the patients with AA, statistical analysis with Student's $t$-test showed a lower $(t=2.3$; d.f. $=144 ; p=0.02)$ mean age in the AA group (30.4 years, $\mathrm{SD}=13.1)$ compared with the control group ( 36.2 years, $\mathrm{SD}=17.2$ ):

Table 2 shows the various modalities of treatment undergone by the AA patients in the past.

\author{
S.M. Recupero \\ S. Abdolrahimzadeh \\ M. De Dominicis \\ R. Mollo \\ Istituto di Oftalmologia \\ Università di Roma \\ 'La Sapienza' \\ Rome, Italy \\ I. Carboni \\ L. Rota \\ S. Calvieri \\ Istituto di Dermatologia \\ Università di Roma \\ 'La Sapienza' \\ Rome, Italy
}

Professor S.M. Recupero Istituto di Oftalmologia

Università di Roma

'La Sapienza'

Policlinico Umberto I

|-00161 Rome, Italy

Tel: +3906490296

Fax: +39064457706

Received: 4 January 1999 Accepted in revised form: 24 June 1999 
Table 1. Associated diseases in the alopecia areata patients

\begin{tabular}{lc}
\hline Pathology & No. of patients (\%) \\
\hline Thyroid pathology & $24(32 \%)$ \\
Atopic dermatitis & $17(23 \%)$ \\
Vitiligo & $2(3 \%)$ \\
Diabetes mellitus & $3(4 \%)$ \\
Neuropsychiatric problems & $15(20 \%)$ \\
Anaemia & $3(4 \%)$ \\
Down's syndrome & $1(1 \%)$ \\
\hline
\end{tabular}

There were no significant relationships between ocular alterations and the duration of illness, age or sex of the patients. Asymptomatic punctate lens opacities were found in $38(51 \%)$ affected patients and $2(3 \%)$ control patients. Furthermore, the opacities were encountered in $14(88 \%), 4(44 \%)$ and $20(40 \%)$ patients with alopecia universalis, totalis and areata, respectively (Table 3). Details of the location of the lens opacities are shown in Table 4. The fundus alterations were, in the majority of cases, at the extreme periphery of the retina and these are detailed in Table 5. They were found in 31 $(41 \%)$ patients with AA and in $16(23 \%)$ control patients. There was no difference in their prevalence in the three forms of AA.

\section{Discussion}

There is a high frequency of ocular alterations in AA, but the first to be reported was the development of cataract, which is, nevertheless, quite rare. ${ }^{2}$ Two other reports were subsequently published. ${ }^{3,4}$ However, in these cases there could have been other possible causes of cataract: long-standing atopic dermatitis, long-term systemic corticosteroid therapy and vitiligo. Also, patients had reached their middle to advanced years of life where the association may be questionable.

Our study did not reveal any cases of cataract in the AA patients but we found a prevalence of symptomless punctate lens opacities which was significantly higher than in the control population $\left(\chi^{2}=41.43\right.$; d.f. $=1$; $p<0.0001)$.

Some authors have reported similar results ${ }^{5}$ while others did not find significant lens changes. ${ }^{6,7}$ This, we believe, is mainly due to the control population taken into consideration in the various reports, which in our study was unaffected by other skin, ocular or systemic diseases that could influence the outcome of the results. We did not find any association with the duration of illness but there was a higher prevalence of lens opacities in alopecia universalis compared with the totalis and areata forms $\left(\chi^{2}=11.10 ;\right.$ d.f. $\left.=2 ; p=0.0038\right)$.

Table 2. Treatment in the alopecia areata patients

\begin{tabular}{lcc}
\hline Treatment & $\begin{array}{c}\text { Total no. of } \\
\text { patients }\end{array}$ & $\begin{array}{c}\text { No. of patients with } \\
\text { punctate lens } \\
\text { opacities (\%) }\end{array}$ \\
\hline Systemic corticosteroids & 22 & $11(50 \%)$ \\
Local (scalp) treatment & 56 & $21(38 \%)$ \\
Cyclosporine & 7 & $3(43 \%)$ \\
PUVA & 4 & $1(25 \%)$ \\
\hline
\end{tabular}

Table 3. Details of alopecia areata and control patients and prevalence of lens opacities

\begin{tabular}{lccc}
\hline Diagnosis & $\begin{array}{c}\text { No. of } \\
\text { patients }\end{array}$ & M/F & $\begin{array}{c}\text { Lens changes } \\
\text { present }\end{array}$ \\
\hline Alopecia universalis & 16 & $9 / 7$ & $14(88 \%)$ \\
Alopecia totalis & 9 & $1 / 8$ & $4(44 \%)$ \\
Alopecia areata & 50 & $15 / 35$ & $20(40 \%)$ \\
Total AA patients & 75 & $25 / 50$ & $\mathbf{3 8 ( 5 1 \% )}$ \\
Control patients & $\mathbf{7 0}$ & $\mathbf{2 5 / 4 5}$ & $\mathbf{2 ( 3 \% )}$ \\
\hline
\end{tabular}

In our group of AA patients 17 also suffered from atopic dermatitis, 8 of whom presented punctate lens opacities. Cataract associated with atopic dermatitis is characteristic; it nearly always begins in the posterior subcapsular area near the pole, growing forward and peripherally in a successive process occurring in the anterior subcapsular zone. It has been reported in approximately $8-10 \%$ of patients with an average age of 22-25.5 years. ${ }^{10,11}$ Furthermore, in the description of the patients studied by Cowan and Klauder ${ }^{10}$ 'tiny, dotlike or punctate' opacities were described in about $10 \%$ of eyes. These opacities do not seem to be clinically different from the punctate opacities observed in AA patients, and therefore an association with atopic dermatitis cannot be strictly ruled out. Thyroid pathology with the presence of anti-thyroid antibodies was observed in 24 patients, 9 of whom had punctate lens opacities. The association of AA with autoantibodies, especially those against thyroid components, has been widely discussed in view of the debated autoimmune pathogenesis of AA. ${ }^{12-16}$ Indeed, a recent article by Gilhar et al.,' has provided strong evidence that the disease has an autoimmune pathogenesis. Its possible association with thyroid pathology needs to be clarified.

The history regarding previous systemic corticosteroid treatment was not reported with precision by the patients with AA. The majority did not have any documentation of previous consultations in various medical settings. There had been previous therapy with steroids but this was mainly local (scalp) applications and in some cases patients claimed that systemic steroids had been administered for short periods (not exceeding 6 months). The treatment protocol administered at the dermatology clinic in 22 patients consisted of deflazacort $15 \mathrm{mg}$ t.i.d. and the ophthalmic examination was performed at a maximum of 1 month from the initiation of this treatment. We encountered symptomless punctate opacities in 11 patients undergoing this therapy. It has been demonstrated that patients receiving $16 \mathrm{mg}$ of prednisone or its equivalent daily for over a year are extremely likely to develop posterior subcapsular cataracts. ${ }^{17,18}$ To our knowledge, the patients in our study had not received this dose of steroids and the duration of treatment had also been less than 6 months. Furthermore, it can be argued that the symptomless punctate opacities seen in our patients were not cataracts arising after steroid treatment as described in the literature. The punctate lens opacities were located in the posterior subcapsular area in only 5 patients and again 


\begin{tabular}{lcccc}
\hline & & & \multicolumn{2}{c}{ Type of lens opacities (no. of patients) } \\
\cline { 3 - 5 } Diagnosis & No. of patients & PAC & PPC & PN \\
\hline Alopecia universalis & 14 & 5 & 6 & 2 \\
Alopecia totalis & 4 & 1 & 0 & 1 \\
Alopecia areata & 20 & 3 & $\mathbf{1}$ & $\mathbf{1}$ \\
Total AA patients & $\mathbf{3 8}$ & $\mathbf{9}$ & $\mathbf{7}$ & $\mathbf{1}$ \\
Control patients & $\mathbf{2}$ & & $\mathbf{1}$ \\
\hline
\end{tabular}

PAC, punctate anterior cortical opacities; PPC, punctate posterior cortical opacities; PN, punctate nuclear opacities; PPSC, punctate posterior subcapsular opacities.

Table 5. Details of fundus abnormalities in alopecia areata and control patients

\begin{tabular}{|c|c|c|c|c|c|c|c|c|c|c|}
\hline & \multirow{2}{*}{$\begin{array}{l}\text { No. of } \\
\text { paients }\end{array}$} & \multicolumn{9}{|c|}{ Fundus abnormalities } \\
\hline & & PC & PD & PVA & $\mathrm{CD}$ & LD & STD & WWP & $\mathrm{RH}$ & Total \\
\hline Alopecia universalis & 16 & 2 & 3 & & 2 & & 1 & & & $7(43 \%)$ \\
\hline Alopecia totalis & 9 & & & 1 & 2 & & 1 & 1 & & $5(55 \%)$ \\
\hline Alopecia areata & 50 & & 6 & 2 & 3 & 1 & 1 & 3 & 2 & $19(38 \%)$ \\
\hline Total AA patients & 75 & 2 & 9 & 3 & 7 & 1 & 3 & 4 & 2 & $31(41 \%)$ \\
\hline Control patients & 70 & 2 & 2 & 2 & 1 & 1 & 3 & 4 & 1 & $16(23 \%)$ \\
\hline
\end{tabular}

PC, pigmentary clumping; PD, paving-stone degeneration; PVA, paravascular adherence; CD, cystic degeneration; LD, lattice degeneration; STD, snail-track degeneration; WWP, white without pressure; RH, retinal hole.

these were symptomless. Furthermore, these changes were also observed in patients who had not received any systemic cortisone treatment. Therefore, we do not believe that these lens alterations can be attributed to this type of treatment. There are no reports in the literature regarding cataracts after local steroid treatment. The origin of these opacities is not clear. However, lens changes are not infrequent in the course of cutaneous disorders and this association is not surprising as the skin and lens have a common embryonic origin from the ectoderm.

There have been various reports on retinal changes occurring in AA patients. ${ }^{5,8,9}$ Studies performed with fluorescein angiography (FAG) of the fundus showed a significantly higher frequency of these alterations in AA patients compared with controls. ${ }^{5,9}$ Furthermore, in those undergoing electroretinography (ERG) and electrooculography (EOG) the ERG was normal but Arden's ratio (calculated from the EOG) was significantly depressed in AA patients. Therefore, it was postulated that the normal photoreceptor function evidenced by ERG and the depressed EOG values could suggest retinal pigment epithelial dysfunction. ${ }^{8}$ The hypothesis that there is some type of pigment defect in AA has been proposed by some authors. $5,8,9,19$ The retinal pigment epithelium could be primarily involved due to alterations of melanocytes or its involvement could be a consequence of a secondary insult. AA is an autoimmune disease with strong evidence for the role of $\mathrm{T}$ lymphocyte activity directed against components of hair follicles. ${ }^{14-16}$ This component could be the melanocytes, as clinically shown by damage to the hair and retinal pigment epithelium.

In our study we found that patients with AA have a prevalence of retinal alterations which is statistically significant compared with controls $\left(\chi^{2}=5.64 ;\right.$ d.f. $=1$, $p=0.0175)$. However, there was no statistically significant difference between the three forms of AA $\left(\chi^{2}=1.02 ;\right.$ d.f. $\left.=2 ; p=0.009\right)$. In our patients we observed a variety of retinal changes; these were at the periphery and in some cases were of the rhegmatogenous type with involvement of the retinal pigment epithelium. We did not find retinal alterations at the posterior pole or in the pre-equatorial zone and we cannot formulate any hypotheses as to the cause of these retinal changes. We did not perform FAG for a number of reasons: the lesions observed were in the peripheral retina; to achieve statistically significant results the control population would have had to undergo FAG; and invasive diagnostic procedures could cause further anxiety in AA patients who already have a tendency to neuropsychiatric problems. ${ }^{4}$ Indeed, there were 15 patients $(20 \%)$ in our study who presented various types of neuropsychiatric problems ranging from schizophrenia to minor depression.

We are by no means the first to study AA patients but most previous reports have appeared in the dermatological literature. We can conclude that the high prevalence of symptomless punctate opacities of the lens and peripheral retina alterations, not particularly emphasised by other authors, make ophthalmological examinations advisable in AA patients.

\section{References}

1. Gilhar A, Ulmann Y, Berkutzi A, et al. Autoimmune hair loss (alopecia areata) transferred by $\mathrm{T}$ lymphocytes to human scalp explants on SCID mice. J Clin Invest 1998;101:62-7.

2. Papastratigakis $C$. Un nouveau syndrome dystrophique juvenile: alopécie totale associée à la cataracte et a des alterations onguéales. Paris Med 1922;45:475-6.

3. Trenel M, Prieur M. Alopécie congénitale familiale héréditaire avec cataracte précoce. Rev Neurol 1930;2:561-5.

4. Muller SA, Brunsting LA. Cataracts associated with dermatologic disorders. Arch Dermatol 1963;88:330-9. 
5. Tosti A, Colombati S, Caponeri GM, Ciliberti C, Tosti G, Bosi M. Ocular abnormalities occurring with alopecia areata. Dermatologica 1985;170:69-73.

6. Summerly R, Watson DM, Monckton PW. Alopecia areata and cataract. Arch Dermatol 1966;93:411-2.

7. Bianchi PE, Malvezzi F, Stringa M, Orecchia G. Lens opacities occurring in alopecia areata. Ann Ottalmol Clin Ocul 1988;114:129-32.

8. Tosti A, Colombati S, De Padova MP, Guidelli Guidi S, Tosti $\mathrm{G}$, Maccolini E. Retinal pigment epithelium function in alopecia areata. J Invest Dermatol 1986;86:553-5.

9. Maccolini E, Zotti CA, Tosti G, Colombati S, Schiavi L. Anomalie dell'epitelio pigmentato retinico in corso di alopecia areata: aspetti elettrofunzionali e fluorangiografici. Boll Ocul 1993;72:935-43.

10. Cowan A, Klauder JV. Frequency of occurrence of cataract in atopic dermatitis. Arch Ophthalmol 1950;43:759-68.

11. Brunsting LA. Atopic dermatitis (disseminated neurodermatitis) of young adults: analysis of precipitating factors in one hundred and one cases and report of ten cases with associated juvenile cataract. Arch Dermatol Syphilol 1936;34:935-57.
12. Friedmann PS. Alopecia areata and auto-immunity. Br J Ophthalmol 1981;105:153-7.

13. Cochran REI, Thomson J, MacSween RNM. An auto-immune profile in alopecia totalis and diffuse alopecia. Br J Dermatol 1976;95:61-6.

14. Kern F, Hoffmann WH, Hambrick GW, Blizzard RM. Alopecia areata: immunologic studies and treatment with prednisone. Arch Dermatol 1973;107:407-12.

15. Main RA, Robbie RB, Gray RS, Donald D, Horne GHW. Smooth muscle antibodies and alopecia areata. $\mathrm{Br} J$ Dermatol 1975;92:389-93.

16. Editorial. Alopecia areata: an autoimmune disease? Lancet 1984;1:1335-6.

17. Oglesby RB, Black RL, von Sallmann L, Bunim JJ. Cataracts in patients with rheumatic diseases treated with corticosteroids: description and differential diagnosis. Arch Ophthalmol 1961;66:97-101.

18. Oglesby RB, Black RL, von Sallmann L, Bunim JJ. Cataracts in patients with rheumatic diseases treated with corticosteroids: further observations. Arch Ophthalmol 1961;66:41-6.

19. Brown AC, Pollard ZF, Jarret WH. Ocular and testicular abnormalities in alopecia areata. Arch Dermatol 1982;118:546-54. 